\title{
Spontaneous ruptured of splenic artery aneurysm: Another reason for fatal syncope and shock(Case report and literature review)
}

\section{Feng Yuan}

Emergency Department of Changsha Central Hospital Affiliated to Nanhua University

\section{Liudang He}

Emergency Department of Changsha Central Hospital Affiliated to Nanhua University

\section{Zhengbin Yao}

Emergency Department of Changsha Central Hospital Affiliated to Nanhua University

\section{Yong Long}

Emergency Department of Changsha Central Hospital Affiliated to Nanhua University

Shugen Xu ( $\nabla$ xushugeng633@163.com)

Changsha Central Hospital Affiliated to Nanhua University https://orcid.org/0000-0002-7113-4925

\section{Case report}

Keywords: splenic artery aneurysm, syncope, shock, POCUS

Posted Date: March 2nd, 2021

DOl: https://doi.org/10.21203/rs.3.rs-251955/v1

License: (9) This work is licensed under a Creative Commons Attribution 4.0 International License. Read Full License 


\section{Abstract}

Background: Splenic artery aneurysm(SAA) is a rare condition, however, it is one of the most common intra-abdominal aneurysm. In the emergency department, due to an uncommon cause of shock and syncope in SAA, it poses a great diagnostic challenge for emergency physicians. Here we reported the case of a spontaneous ruptured of splenic artery aneurysm, which was detected by point-of-care ultrasound (POCUS), in a 47-year-old man with syncope and shock. The diagnosis was further confirmed with a computed tomography scan and surgery.

Case presentation: A 47-year-old man presented to the emergency department(ED) for syncope and shock. As he had unstable hemodynamic so we gave him fluid resuscitation and point-of-care ultrasound (POCUS), free intraperitoneal fluid was identified on ultrasound, then a diagnostic abdominal paracentesis was performed and hemorrhagic ascites were identified. The rare but life-threatening diagnosis of spontaneous ruptured of splenic artery aneurysm was confirmed by contrast-enhanced CT and surgery.

Conclusions: Spontaneous splenic artery aneurysm ruptured is a rare fatal condition needs immediately diagnosis and management to achieve a favorable outcome. Though there is no risk factors, emergency physicians should considered SAA in the differential diagnosis of sudden collapse. As an emergency physicians, it is very important to be master first aid skills such as POCUS.

\section{Background}

Splenic artery aneurysm (SAA) is a rare potentially fatal condition, and the incidence varies from 0.1 to $10.4 \%$ in the general population ${ }^{[1]}$, It is the third most common intra-abdominal aneurysm followed by aortic and iliac arteries aneurysms ${ }^{[2]}$. With the potential risk for rupture and life threatening hemorrhage shock, the mortality rate of ruptured SAA is $10-25 \%$ in non-pregnant patient and up to $70 \%$ during pregnancy ${ }^{[4]}$. With the advances made of radiologic studies and increased aging population, the diagnoses of SAA are increasing. It is four times more common in females compared to males from the study by Dave SP et.al ${ }^{[4]}$, but it seems no different in the study of Hamid et al[5]. Although the pathogenesis is not fully understood, the risk factors include pregnancy, portal hypertension, splenomegaly, medial fibroplasia, liver cirrhosis, liver transplantation, degenerative atherosclerosis, pancreatic pseudocyst, polyarteritis nodosa, vasculitis and congenital anomalies affecting the arteries of the foregut ${ }^{[6-7]}$. In our case of spontaneous splenic artery aneurysm rupture, it seems without any previously known risk factor.

\section{Case Presentation}

A 47-year-old male was admitted to our emergency department by emergency medical services (EMS)due to a sudden syncope and shock $30 \mathrm{mins}$ after lunch. There was no associated symptoms nor a history of any disease or trauma. He was conscious when on admission囚and just felt dizzy and tired with cold 
sweat. Physical examinations: heart beats 132 per min, blood pressure 82/51 $\mathrm{mmHg} \otimes$ respiration rate 20 per min, and transcutaneous oxygen saturation $99 \%$. Neurological examination showed no abnormal findings, also no meaningful found in chest and heart examination, abdomen was soft, epigastric abdominal mild general tenderness, but no rebound tenderness; liver and spleen were impalpable. We thought the syncope was the result of hypovolemic shock, so we gave him fluid resuscitation and pointof-care ultrasound (POCUS), free intraperitoneal fluid was identified on ultrasound, then a diagnostic abdominal paracentesis was performed and hemorrhagic ascites were identified. A contrast-enhanced CT was performed immediately. CT angiogram showed large amount of free intraperitoneal fluid and a splenic artery aneurysm with intravenous contrast extravasation consisting of intra peritoneal hemorrhage(Figs. 1 and 2). After that, an emergency operation was implemented. A midline laparotomy incision was performed, and a total amount of $3 \mathrm{~L}$ blood were evacuated. The splenic artery was ligated proximally, followed by a splenectomy. The patient was transferred to the ICU for unstable hemodynamic after surgery, but was transferred to general ward next day. The patient had recovered and discharged home on the 7th postoperative day. During the one-year follow-up, the patient lived well without complications. The histopathology report confirmed the diagnosis of a true splenic artery aneurysm(Figs.3 and 4).

\section{Discussion}

SAA includes true aneurysms and pseudoaneurysms. The different between them is all three layers of the vessel wall where the true aneurysm located are thinned and dilated, but the pseudoaneurysms resulting from a tear in the vessel wall which subsequent formation of a peri-arterial hematoma.

Pseudoaneurysms are usually posttraumatic or the result of a local surrounding inflammation ${ }^{[8]}$. The pseudoaneurysms which lacks one or more of the layers of the vessel wall, leads to making it weaker and more susceptible to rupture.

The majority of patients with SAA are asymptomatic, which make it usually are diagnosed incidentally ${ }^{[9]}$. About 20\%SAA patients have nonspecific symptoms such as abdominal pain in the epigastrium or left upper quadrant, chest pain, anorexia, nausea or vomiting. Though the pathogenesis is not fully understood, some risk factors for rupture of the aneurysms are confirmed, include pregnancy, a diameter greater than $2 \mathrm{~cm}$, portal hypertension, development of symptoms, and liver transplantation et.al ${ }^{[10,11]}$.

Splenic artery aneurysm rupture resulted in an active intraabdominal bleed and hemorrhagic shock, which could be fatal if not treated timely. It will be much more serious for pregnancy patient, to whom the mortality rate can reach as high as $75 \%{ }^{[12]}$. Even worse, some studies showed that about $95 \%$ of splenic artery aneurysm ruptures occurred in pregnancy ${ }^{[13]}$. For diagnosis of ruptured SAA, contrast-enhanced $\mathrm{CT}, \mathrm{MRI}$ and MRA were more sensitive and have the advantage of providing three-dimensional compared to conventional ultrasound ${ }^{[14]}$, The gold standard for diagnosis SAA is digital subtraction angiography (DSA), which can provide the precise location of the aneurysm, assess Collateral branches, locate the source of bleeding and document or exclude other visceral aneurysms simultaneously ${ }^{[4]}$. 
The basic managements of ruptured SAA mainly include fluid resuscitation and hemodynamic support, but no matter the hemodynamic stable or not, an urgency operation is needed. More, it is suggest that all

symptomatic splenic artery aneurysms should be treated as a matter of urgency ${ }^{[15]}$. A range of therapeutic options are available to deal with SAA. Open surgical approaches is the first choice for ruptured SAA, but the stenting or coil embolization can also be used for the both ruptured and asymptomatic $S A A^{[8]}$. Open surgical approaches may include splenectomy with removal of the aneurysm, proximal and distal splenic artery ligation with or without resection of the aneurysm and transaneurysmal arterial ligation ${ }^{[16]}$, which depend on patient's profile. Partial splenectomy are performed in order to preserve immune function. However some evidence suggests ligation or embolization of the splenic artery also impairs splenic function despite preservation of the organ ${ }^{[17]}$. The endovascular treatment usually be operated among the patients who are not candidate for surgery or hemodynamic stability.

In our case, the patient was confirmed to be a true splenic aneurysm after surgery, but he had no risk factors, and before the rupture, the patient had no symptoms, even after the splenic aneurysm ruptured, the patient's symptoms were not typical, this made our timely diagnosis and treatment more difficult, although the patient finally recovered through surgery, the diagnosis process was full of challenges, and even a little hesitation may be fatal to the patient. From this case, we can also see that the applications such as point-of-care ultrasound (POCUS) or FAST(Focused Assessment with Sonography for Trauma) in the emergency department can provide timely and efficient diagnosis and treatment for doctors and significantly improve the prognosis of patients, more importantly, in the emergency department, patients with unclear diagnosis need to be treated and differentially diagnosed according to procedures or guides.

\section{Conclusions}

Splenic artery aneurysm ruptured is a rare and fatal condition, not treatment timely often imperil the patient lives. For ruptured SAA, the emergency department is the first place when they arrive hospital, so when patients presenting with abdominal pain, syncope and signs of hypovolemia, the diagnosis of ruptured SAA should be considered for emergency physicians. Prompt treatment is detrimental to patient survival; it emphasizes the role of emergency diagnosis and treatment process and easily available diagnostic modalities such as POCUS.

\section{Abbreviations}

SAA: Splenic artery aneurysm; POCUS: Point-of-care ultrasound; ED: Emergency department; FAST: Focused Assessment with Sonography for Trauma; EMS: Emergency medical services; DSA: Digital subtraction angiography; EICU: Emergency intensive care unit

\section{Declarations}


Not applicable.

\section{Authors contributions}

Xu receives and initially treats patients in the emergency department, Long, He and Yuan give patients emergency surgery, and Yao gives patients postoperative treatment and follow-up. Xu and Yuan wrote and revised the paper.

\section{Funding}

This study did not receive any specific grants from any funding agencies in the public, commercial, and non-profit sectors.

\section{Availability of data and materials}

Not applicable.

\section{Ethics approval and consent to participate}

Since this is a case report, approval from the local ethical committee is not necessary.

\section{Consent for publication}

The patient signed consent for publication of data (including individual details and images).

\section{Competing interests}

The authors declare that they have no competing interests.

\section{Author details}

${ }^{\square}$ Emergency Department of Changsha Central Hospital Affiliated to Nanhua University, Changsha, Hunan Province, China.

\section{References}

1. Messina LM, Shanley CJ. Visceral artery aneurysms. Surg Clin North Am 1997;77(2):425-42.

2. Al-Habbal Y, Christophi C, Muralidharan V. Aneurysms of the splenic artery - a review. Surg J R Coll Surg Edinb Irel 2010;8(4):223-31.

3. Manian U, Badri H, Coyne P, Nice C, Ashour H, Bhattacharya V. Endovascular Treatment of a Ruptured Splenic Artery Aneurysm using Amplatzer(®) Vascular Plug. Int J Biomed Sci. 2009 Mar;5(1):81-4.

4. Dave SP, Reis ED, Hossain A, Taub PJ, Kerstein MD, Hollier LH. Splenic artery aneurysm in the 1990s. Ann Vasc Surg 2000;14(3):223-9. 
5. Hamid HKS, Suliman AEA, Piffaretti G, Spiliopoulos S, Tetreau R, Tozzi M, Pulli R. A systematic review on clinical features and management of true giant splenic artery aneurysms. J Vasc Surg. 2020 Mar;71(3):1036-1045.e1.

6. Kim Y, Johna S. Laparoscopic excision of splenic artery aneurysm. J Soc Laparoendosc Surg/Soc Laparoendosc Surg: JSLS 2013;17(1):132-4.

7. Annicchiarico BE, Avolio AW, Caracciolo G, Barbaro B, Di Stasi C, Agnes S, et al. Late development of splenic artery aneurysm after orthotopic liver transplantation: a case report. Transplant Proc 2009;41(4):1383-5.

8. Cordova AC, Sumpio BE. Visceral artery aneurysms and pseudoaneurysms-should they all be managed by endovascular techniques? Ann Vasc Dis 2013;6(4):687-93.

9. Sarikaya S, Ekci B, Aktas C, Cetin A, Ay D, Demirag A. A rare clinic presentation of abdominal pain: rupture of splenic artery aneurysm: a case report. Cases J 2009;2:148.

10. de Perrot M, Buhler L, Deleaval J, Borisch B, Mentha G, Morel P. Management of true aneurysms of the splenic artery. Am J Surg 1998;175(6):466-8.

11. Hallett JW Jr. Splenic artery aneurysms. Semin Vasc Surg. 1995 Dec;8(4):321-6.

12. Nanez L, Knowles M, Modrall JG, Valentine RJ. Ruptured splenic artery aneurysms are exceedingly rare in pregnant women. J Vasc Surg. 2014 Dec;60(6):1520-3.

13. de Csepel J, Quinn T, Gagner M. Laparoscopic exclusion of a splenic artery aneurysm using a lateral approach permits preservation of the spleen. Surg Laparosc Endosc Percutan Tech 2001;11(3):2214.

14. Sadat U, Dar O, Walsh S, Varty K. Splenic artery aneurysms in pregnancy-a systematic review. Int J Surg 2008;6(3):261-5.

15. Trastek VF, Pairolero PC, Joyce JW, Hollier LH, Bernatz PE. Splenic artery aneurysms. Surgery. 1982 Jun;91(6):694-9.

16. Arca MJ, Gagner M, Heniford BT, Sullivan TM, Beven EG.Splenic artery aneurysms: methods of laparoscopic repair. J Vasc Surg 1999;30(1):184-8.

17. Nincheri Kunz M, Pantalone D, Borri A, Paolucci R, Pernice LM, Taruffi F, Andreoli F. Management of true splenic artery aneurysms. Two case reports and review of the literature. Minerva Chir. 2003 Apr;58(2):247-56.

\section{Figures}




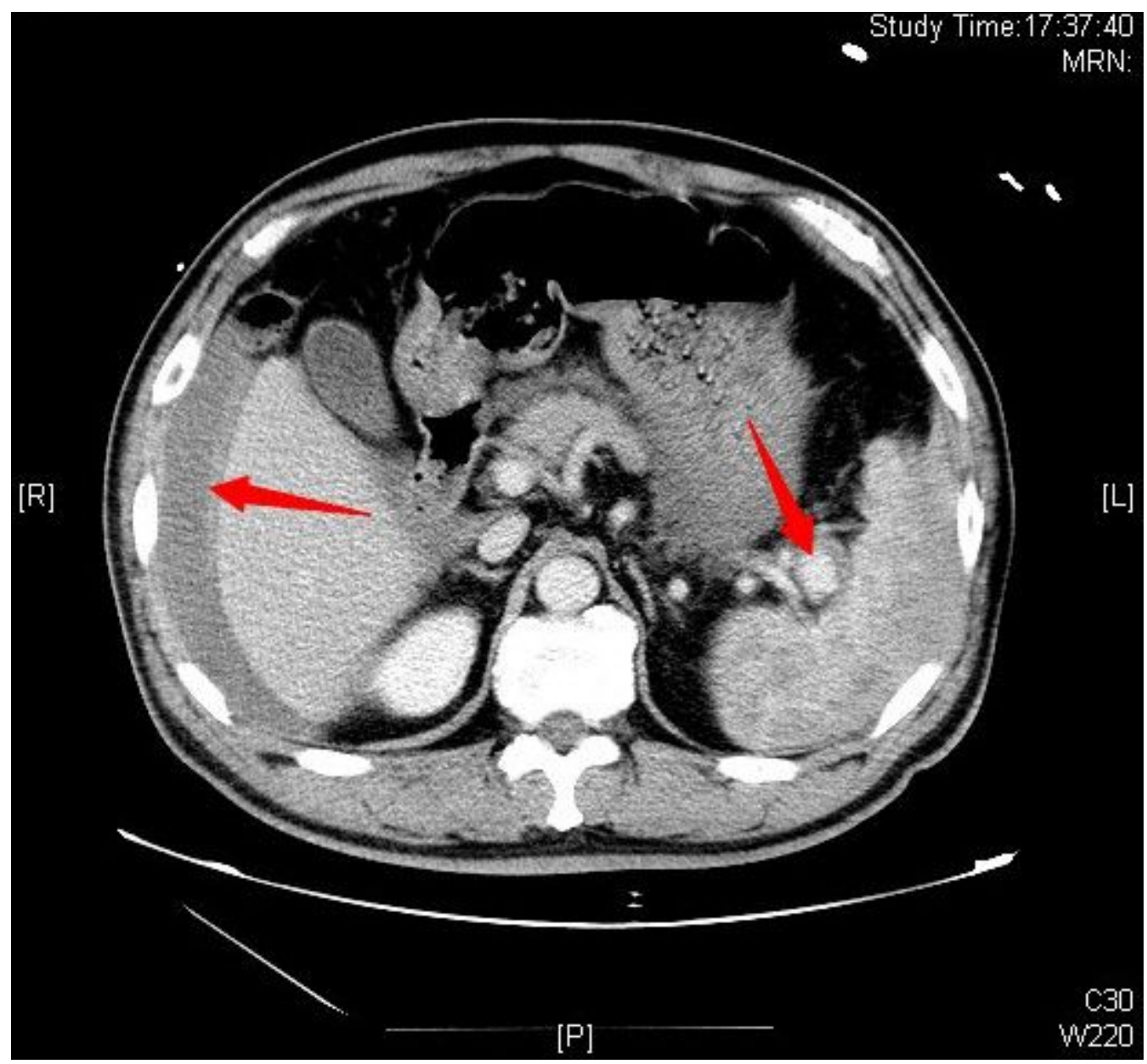

Figure 1

Contrast-enhanced CT of the abdomen showed perihepatic hemorrhage and splenic aneurysm(arrows) 


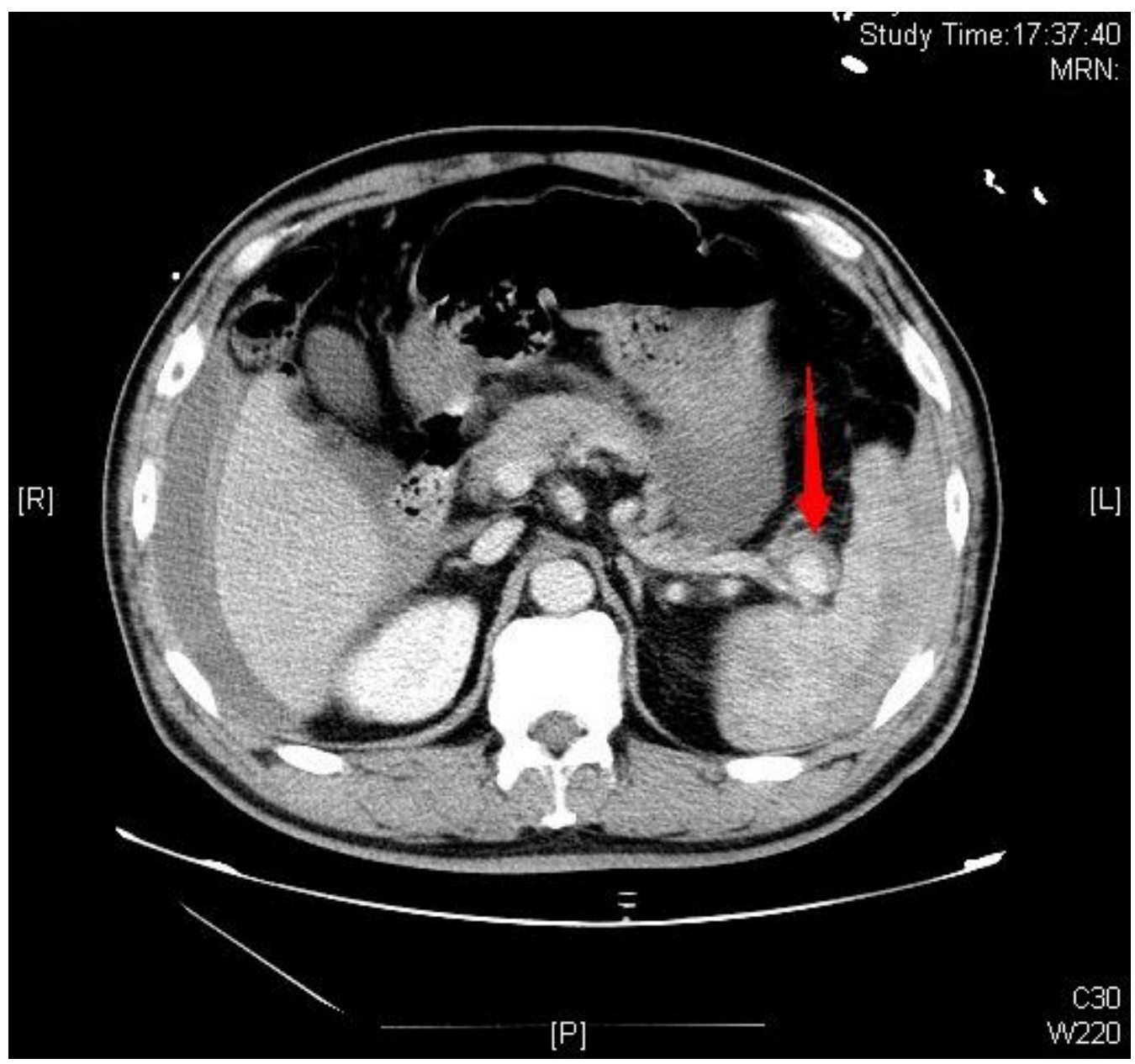

Figure 2

Contrast-enhanced CT of the abdomen showed Contrast agent extravasation and ruptured splenic aneurysm (arrow) 


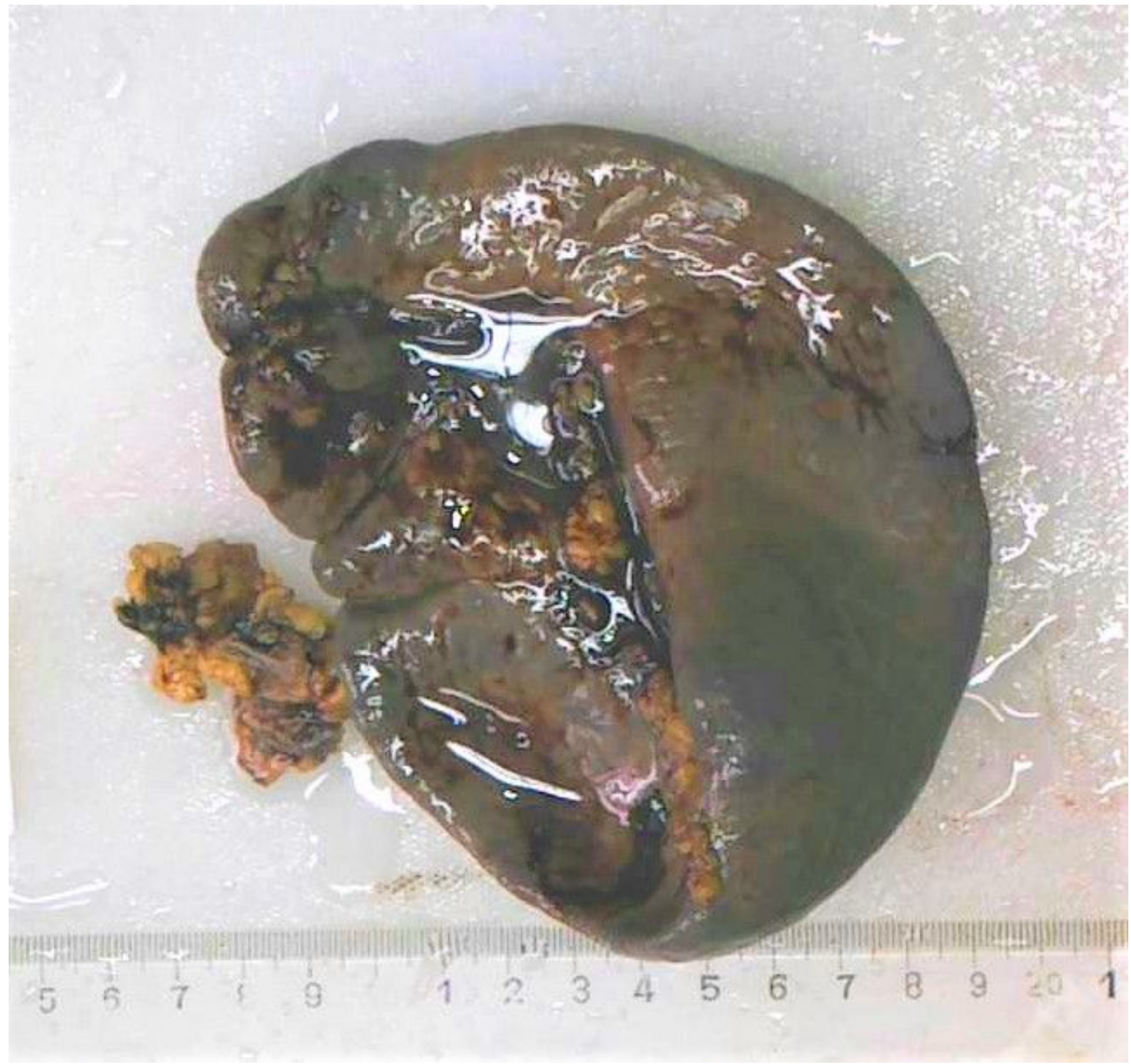

\section{Figure 3}

The histopathology report confirmed the diagnosis of a true splenic artery aneurysm 
标本名称: 腹腔 (脾脏)

临床诊断: 腹腔出血查因

特征图像:
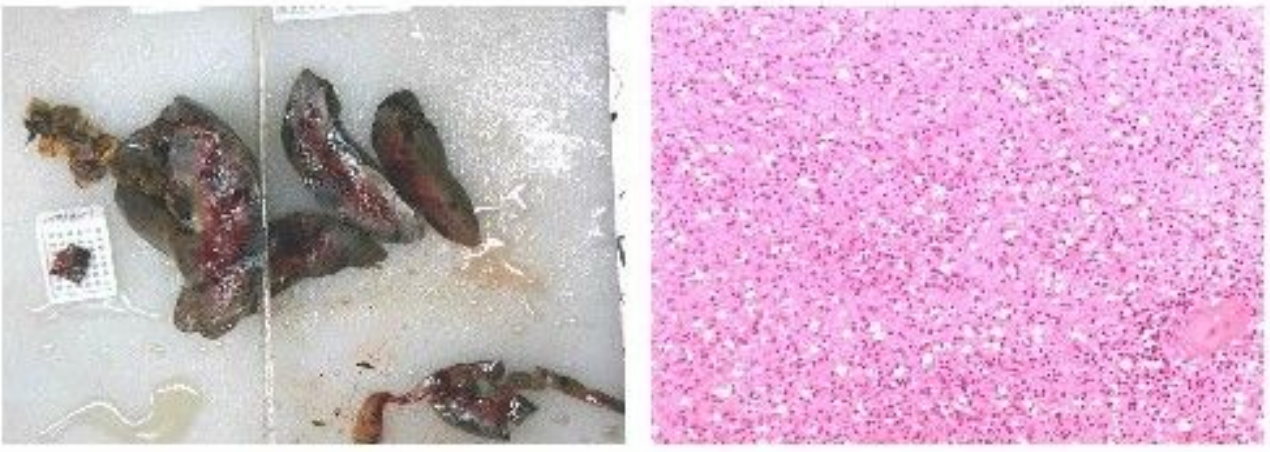

肉眼所见:

脾脏一个, 大小 $13 \times 9 \times 6.5 \mathrm{~cm}$, 表面较光滑, 灰白灰褐, 脾门处表面附有血凝垬, 切面脾实质 内见弥漫点片状出血, 呈地图状分布, 灰红灰褐。另有脂肪样游离组织一块, 大小

$4 \times 1.5 \times 0.8 \mathrm{~cm}$, 灰黄灰褐, 切面可见血管结构, 管径约 $0.3 \mathrm{~cm}$ 。

病理诊断:

(脾脏) 符合动脉瘤破裂并脾脏内出血, 并见脾实质内中央动脉玻变硬化。

\section{Figure 4}

The histopathology report confirmed the diagnosis of a true splenic artery aneurysm 